\section{Estimation of Pyrethrum Flower Number Using Digital Imagery}

\author{
Jason B. Scott ${ }^{1,4}$, David H. Gent ${ }^{2}$, Frank S. Hay ${ }^{3}$, \\ and Sarah J. Pethybridge ${ }^{3}$
}

ADDITIONAL INDEX WORDS. remote sensing, sample size, spatial variability, yield estimation, image analysis, Tanacetum cinerarifolium

\begin{abstract}
SuMMARY. Flower number is the primary determinant of yield in pyrethrum (Tanacetum cineariffolium). Traditional estimates of flower numbers use physical harvesting of flowers, which is time consuming, destructive, and complicated. The precision of flower number estimates may be highly influenced by spatial heterogeneity of plant density and vigor. Here, we examined the potential for digital image analysis to enable rapid, nondestructive assessment of flower number. This technique involved removal of pixels with color profiles not typical of the disc florets of pyrethrum. Particle counting was then performed using defined size and shape parameters to estimate flower numbers. Estimates of flower number based on image analyses were correlated with physical harvests of flowers, with estimates representing about an average of $32 \%$ of total flower numbers present within a sampling unit. This relationship was consistent across all observed flower densities. Covariate analysis indicated that occurrences of crop lodging and over mature flower canopies had significant, detrimental effects on system predictions. Pyrethrum flowers were spatially aggregated within fields with the degree of aggregation greatest at the lowest flower densities. Based on modeled flower distributions, eight quadrats $\left(0.49-\mathrm{m}^{2}\right.$ sampling unit) were sufficient to achieve a $\mathrm{CV}$ of 0.1 in a $600-\mathrm{m}^{2}$ plot area in all but the lowest flower densities. The utility of this approach for biomass assessment in pyrethrum and other Compositae is discussed.
\end{abstract}

$\mathrm{P}$ yrethrum is a perennial, herbaceous plant grown for the production of pyrethrins in the achenes of flowers (Zito et al., 1983). Pyrethrins have insecticidal properties and are used as the a.i. of many products that may be used in organic agriculture or for pest control in households (Casida and Quistad, 1995). In Australia, pyrethrum is grown on a semiperennial basis with the majority of crops sown by direct seeding in early spring (August). Initiation of flowering stems occurs $\approx 14$ months later (October). Flowering is

We thank Craig Palmer for technical assistance in the field and Botanical Resources Australia-Agricultural Services Pty. Ltd. for funding.

Mention of trade names or commercial products in this publication is solely for the purpose of providing specific information and does not imply recommendation or endorsement by the U.S. Department of Agriculture.

${ }^{1}$ Tasmanian Institute of Agriculture, University of Tasmania, P.O. Box 3523, Burnie, Tasmania, 7320 Australia

${ }^{2}$ USDA-ARS, Forage Seed and Cereal Research Unit, and Department of Botany and Plant Pathology, Oregon State University, Corvallis, OR 97331

${ }^{3}$ Section of Plant Pathology and Plant-Microbe Biology, School of Integrative Plant Science, Cornell University, Geneva, NY 14456

${ }^{4}$ Corresponding author. E-mail: Jason.Scott@utas. edu.au. synchronous and begins in early November and continues until harvest 6 to 8 weeks thereafter, when seeds are formed (Macdonald, 1995). The production system is highly mechanized (Pethybridge et al., 2008b) and harvests occur annually for up to 5 years. Australia currently produces $\approx 70 \%$ of the global market for pyrethrins.

Pyrethrin yield is determined by flower biomass and pyrethrin ester content of the biomass. Ester content appears to be controlled predominantly by host genetics ( $\mathrm{Li}$ et al., 2014; Wanjaia, 1990); although nutrient deficiencies may play a role (Salardini et al., 1994b). Flower biomass is typically influenced by environmental, physiological, and disease stresses (Pethybridge et al., 2007, 2008a, 2014; Rao et al., 1983; Salardini et al., 1994a), and is therefore the main focus of agronomic studies. Biomass measurements in pyrethrum have historically been conducted by manual flower harvest involving either physical removal of flowers by hand or mechanical means, such as a motorized hedge trimmer. Harvested flowers are counted and assessed for dry weight (Pethybridge et al., 2008a). Sampling from a representative area is critical to achieving accurate and precise estimates of yield. However, many abiotic and biotic factors influence the density and vigor of the stand, which directly influences flower numbers and therefore yield (Pethybridge et al., 2008b). Previous work has shown that stem height in spring strongly correlated with flower yield (Pethybridge et al., 2008a). However, manual stem measurements are also destructive and time consuming, thus providing no benefit over manual flower harvest. Thus, a primary limiting factor associated with both techniques is that they are highly labor intensive and limit the number of treatments and areas that may be assessed. Moreover, manual harvest is destructive to the sampled area and may cause considerable damage to the surrounding crop. This damage may affect subsequent assessments of regrowth after harvest. Therefore, the ability to estimate flower number as a primary component of yield before harvest via rapid, nondestructive, and reproducible means would be beneficial as a research tool.

Digital image analysis can provide the basis for quantifying and enumerating a range of agronomic traits. Systems based on image analysis offer rapid, nondestructive, and reproducible means of sample analysis (Bechar et al., 1997; Costa and Yang, 2009). The image analysis software, Image J, includes several tools that can be employed for analysis of agronomic traits. ImageJ is freeware provided by the U.S. National Institutes of Health, and is compatible with a variety of computer platforms (Schneider et al., 2012). Numerous areas of study have already employed image analysis and ImageJ software including quantifying root development (Henry et al., 2011; Tajima and Kato, 2013), pollen grain counting (Costa and Yang, 2009), color

\begin{tabular}{llll}
\hline $\begin{array}{l}\text { Units } \\
\begin{array}{l}\text { To convert U.S. to SI, } \\
\text { multiply by }\end{array}\end{array}$ & U.S. unit & SI unit & $\begin{array}{l}\text { To convert SI to U.S., } \\
\text { multiply by }\end{array}$ \\
\hline 0.3048 & $\mathrm{ft}$ & $\mathrm{m}$ & 3.2808 \\
0.0929 & $\mathrm{ft}^{2}$ & $\mathrm{~m}^{2}$ & 10.7639
\end{tabular}


uniformity of turf (Ghali et al., 2012), seed shape of wheat [Triticum aestivum (Williams et al., 2013)], and maize (Zea mays) kernel counting (Severini et al., 2011). The adoption of these systems could be increased if image capturing and processing time and cost were reduced.

The objective of this study was to develop a rapid method for assessing pyrethrum flower number in situ. A secondary objective was to determine the minimum sample number required to produce an accurate estimate of flower number. This is required for the implementation of an image analysis-based technique since spatial variability of flower number impacts minimum sample number.

\section{Materials and methods}

IMAGE COLLECTION AND ANALYSIS. Images of flowers were taken within commercial fields in the first 2 weeks of December when the ray florets were horizontal and before disc floret necrosis. Digital cameras were mounted on a 1.8 -m-tall frame incorporating a horizontal, $0.49-\mathrm{m}^{2}$ square quadrat at $0.9 \mathrm{~m}$ above ground level, which is the approximate terminal height of the flowers. This quadrat was visible within all images and used to define the area for assessment. Cameras were orientated with the lens facing down and perpendicular to the horizontal plane. A cable remote shutter trigger was used for image capture. Except where indicated, all images were collected using a digital camera (Powershot Gl2; Canon Australia, Macquarie Park, NSW, Australia). Images were collected with a fixed aperture size of $\mathrm{f} / 8.0$ and light sensitivity set to ISO 100. Shutter speed was allowed to vary to account for variations in light intensity.

Analysis of images was conducted using Image J $1.47 \mathrm{~g}$ software (Schneider et al., 2012). Before image analysis, preprocessing of images was conducted to standardize orientation and area to be assessed. Image files in jpeg format were opened within ImageJ as red-green-blue (RGB) images. Images were straightened by measuring the angle of deviation of the top edge of the quadrat from parallel to the nearest boundary of the image. Images were then rotated to correct for this deviation using a bilinear interpolation. Following straightening, images were cropped to the boundaries of the quadrat (Fig. 1).

Following preprocessing, images were subject to a sequence of procedures within ImageJ to enable estimation of flower number (Fig. 1). This protocol was automated with a macro that allowed batch estimation for all images present within a single directory. Initially, a color threshold mask was applied to the RGB images based on defined hue, saturation, and brightness (HSB) thresholds. Threshold parameters were determined during system development described below. RGB images were converted to 8-bit (grayscale) images, which were then converted to binary (black and white) images. Particles in binary images were counted using the "Analyze Particles" function. Particles were defined as black pixel clusters resultant from masking of flower disc florets, with size (minimum and maximum pixel area) and shape (circularity) limits determined during the course of system development described below.

System DeVelopment. Fiftyeight images were collected from pyrethrum fields representative of the cultivars and standard production practices for weed, nutrition, and disease management in Tasmania, Australia (Pethybridge et al., 2008b). Before all analyses, images were preprocessed as outlined earlier. Flower numbers within each image were then estimated by a single operator by manually marking and counting flowers with visible disc florets using the "Cell Counter" function of ImageJ.

Following manual estimation of flower numbers by Image J, these images were used to define the HSB, particle size, and shape parameters. First, 10 flowers were arbitrarily selected from each image and the individual hue, saturation, and brightness profiles of the pixels within each selection were recorded separately. Pixel values for each parameter across all individual images were combined into individual data sets and the $1 \%$ and $99 \%$ percentiles of observed values were used to define HSB limits for flower detection.

The initial upper particle limit was determined by manually selecting the largest visible flower within each image and measuring its pixel area.
The maximum pixel area, measured in square pixels $\left(\mathrm{px}^{2}\right)$, obtained across all images was then rounded to the nearest $100 \mathrm{px}^{2}$. Subsequently, images were subject to flower estimation with incremental changes to the lower particle size limit, with minimum area increasing from 0 to $200 \mathrm{px}^{2}$ in $50-\mathrm{px}^{2}$ increments. Initial circularity limits were set at the extremes of 0 and 1 . Estimates of the number of flowers were compared with manually obtained counts, and the lower limit value was selected by comparing the median, interquartile range, and maximal range of observed errors. After the lower limit value was defined, a range of values for the upper size limit were examined at $500-\mathrm{px}^{2}$ increments from 2000 to $5000 \mathrm{px}^{2}$, plus no upper limit. Using these optimal lower and upper limits of the particle size the particle circularity limits were determined. Pyrethrum disc florets are circular, thus the upper circularity limit of 1.0 (a perfect circle) was predefined. Lower limit of circularity was determined by increasing values from 0 (all possible shapes) to 0.5 , in 0.1 increments and comparing the obtained distribution of errors.

After parameter limits were defined, final automated estimates of flower numbers were obtained for each image. Automatic counts were regressed against manual counts for each images using $\mathrm{R}$ version 3.0.2 ( $\mathrm{R}$ Core Team, 2015). The obtained slope and intercept parameters were tested for deviations from 1 and 0 , respectively. In addition, Lin's concordance correlation coefficient $\left[\rho_{\mathrm{c}}\right.$ (Lin, 1989)] and the associated estimates of location shift, scale shift, and corrected bias $\left(\mathrm{C}_{\mathrm{b}}\right)$ were calculated using the epiR package within $\mathrm{R}$ (Stevenson et al., 2015). Ninety-five percent confidence intervals $\{$ bias corrected and accelerated [95\% confidence interval (CI)]\} for each concordance parameter were estimated via bootstrapping using the boot package within R (Canty and Ripley, 2015).

Modeling SAmple size. To examine the number of single images required to reliably estimate flower count, single plots measuring $20 \times$ $30 \mathrm{~m}$ were established in 85 commercial pyrethrum fields during 2010 and 2011. Fifty nonoverlapping images were collected in the first 2 weeks of 


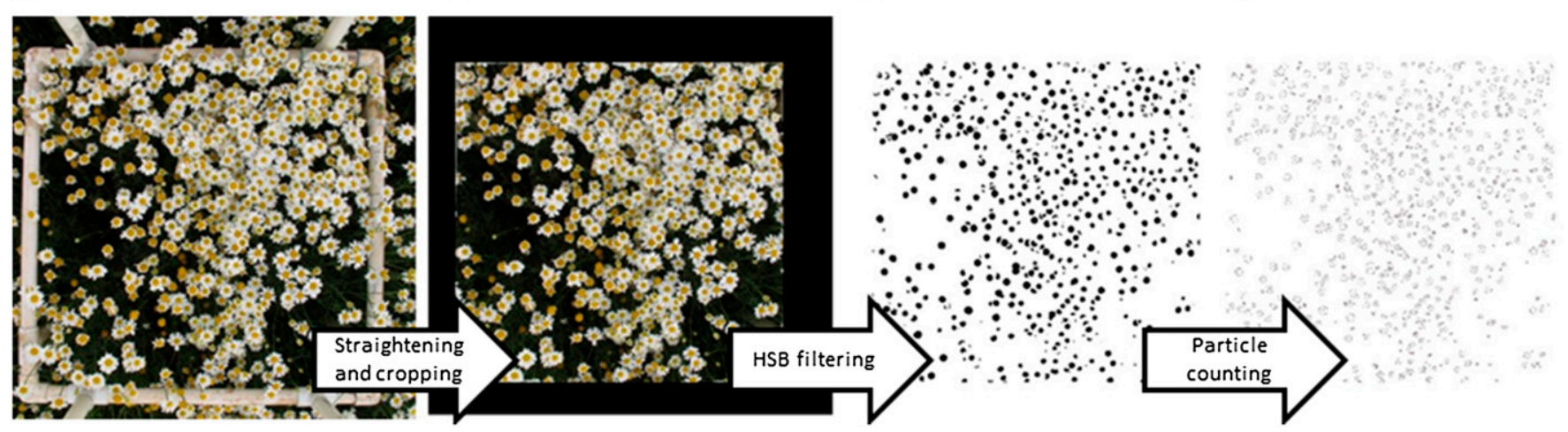

Fig. 1. Images representing the steps involved in capture (A), straightening and cropping (B), color masking based on defined hue, saturation and brightness (HSB) thresholds and conversion to binary (C) to enable definition and counting of particles based on size and shape of the disc florets (D) of pyrethrum.

December from arbitrarily dispersed positions within each of these plots. Flower number per image was estimated by automated processing and used to calculate mean flower count per field. Plots were then ordered by mean flower count and divided into 17 groups, with each group consisting of five plots. From each group, one plot was randomly removed and set aside for model validation. Images from these plots are referred to as model validation plots. The remaining 68 plots were used for modeling sample size as outlined below.

To describe the distribution of the number of flowers in a sample of 50 images within a plot, the Poisson and negative binomial distributions were fit to each data set. Distribution fitting was conducted by maximum likelihood estimation using the MASS package in $\mathrm{R}$ (Venables and Ripley, 2002). The goodness of fit of these distributions was independently tested with $\chi^{2}$ tests. The predictive power of the negative binomial distribution relative to the Poisson distribution was tested using the log-likelihood ratio statistic. In addition, curves based on parameter estimates for each distribution were overlayed on plots of the observed distribution of flower counts of each data set for visual comparison. Taylor's power law was used to examine the relationship between plot mean and variance across all fields (Taylor, 1961).

Based on the results of distribution fitting and Taylor's power law outlined above, the required sample size $(N)$ to achieve a set $\mathrm{CV}$ of means estimates for each plot was determined using the equation:

$$
N=\left(\frac{1}{\bar{F}}+\frac{1}{\hat{k}}\right)\left(\frac{1}{\mathrm{CV}^{2}}\right)
$$

where $\hat{k}$ is the aggregation parameter obtained from fitting the negative binomial distribution to each plot (Madden et al., 2007). Values of $N$ were calculated for each plot for $\mathrm{CV}$ values of $0.05,0.1$, and 0.2 .

The relationship between $N$ and mean flower count $(F)$ was examined by fitting linear $\left[N=\left(a_{\operatorname{lin}}+b_{\operatorname{lin}}\right) F\right]$, exponential $\quad\left(N=a_{\text {exp }} \mathrm{e}^{F b_{\text {cxp }}}\right)$, and power $\left(N=a_{\text {pow }} F^{b_{\text {pow }}}\right)$ models to the data. The linear model was fitted by linear regression, while both the exponential and power modeling were conducted using nonlinear least squares regression. Starting parameters for the exponential model were estimated from the slope $\left(b_{\exp }\right)$ and natural exponent of the intercept $\left(a_{\exp }\right)$ obtained from linear regression of $\ln (N)$ and $F$. Similarly, linear regression of $\log _{10}(N)$ and $\log _{10}(F)$ was used to obtain intercept $\left[=\log _{10}\left(a_{\text {pow }}\right)\right]$ and slope estimates $\left(=b_{\text {pow }}\right)$ to calculate starting parameter estimates for the power model. The relative predictive power of each model was assessed by comparing model deviances.

The sample size (rounded up to an integer) sufficient to achieve a CV of 0.1 or better for $95 \%$ of plots in the modeling development data set, termed $N_{95}$, was calculated. This value was then used to assess the difference between actual and estimated flower counts in the validation plots and the $\mathrm{CV}$. For each validation plot, the mean of all 50 images available for flower counts was calculated and considered the true flower count. Bootstrap estimates of mean flower count were obtained by randomly selecting $\mathrm{N}_{95}$ samples, with replacement after each selection, from within each plot. One thousand bootstrap replicates were obtained for each data set to calculate estimated mean flower count and achieved cv. Within each bootstrap replicate, estimated flower count was regressed on true flower count to obtain bootstrapped estimates of the slope and intercept parameters of this relationship. From all replicates, median and 95\% CI were obtained for estimated flower count and achieved $\mathrm{CV}$ for each individual plot. Median estimated flower count was regressed against true flower count for the slope and intercept parameters, and coefficient of determination obtained. The significance of deviations from 0 (intercept) or 1 (slope) for regressions was tested by $t$ tests. Concordance correlation parameters were calculated as described.

System validation. Within five commercial pyrethrum fields in December in each of 3 years (2011-13), 10 quadrats were demarcated for sampling. At each sampling unit once the camera frame was positioned, boundaries were fixed by the placement of solid plywood barriers on each side of the quadrat, from ground level to $1 \mathrm{~m}$ in height. Subsequently, one digital image of the crop canopy was taken using the standardized camera settings. Images in 2011 and 2012 were captured using a Powershot G12 digital camera, while in 2013 a Powershot G1X (Canon Australia) was used. Following image 
capture, all pyrethrum flowers within the quadrat were hand harvested and counted manually.

Images were first examined manually and scored for the presence or absence of factors during image collection that could potentially influence automated flower counts. The sources of potential error considered here fell into two broad categories, crop development and environmental conditions during capture. Crop development factors included crop lodging (Lodging) and crop maturity (Maturity) with over mature crops defined as when the ray florets collapse and abscise [flower maturity stage 6 (Pethybridge et al., 2005)]. Environmental conditions that were deemed potential sources of error were high contrast conditions resulting in shading (Shadows) and free water due to rain (Rain). Manual counts of flowers visible within each image were then obtained using the standard protocol. Automated estimates of flower number were obtained using the HSB, particle size, and particle shape limits defined during system development. To account for differences in resolution and sensor size between the camera models, images collected using the Powershot GIX were analyzed using pixel area limits that were inflated to $1.5 \times$ those collected using the Powershot Gl2.

Automated estimates were then compared with manual counts of flowers within images and handsampled flower counts using linear regression and concordance correlation parameter estimation. In addition, these relationships between manual and automated estimates were compared with those obtained during model development.

Covariate analysis was used to examine the influence of potential sources of error on the relationship between hand-sampled counts and automated estimates. The linear relationship between these parameters was designated the null model. All factors were then included separately, first as an intercept term and then as a slope interaction term within the null model. If inclusion of a factor significantly $(P<0.05)$ reduced the sum of squares error (SSE) of this model relative to the null model that factor was considered significant. Differences in SSE between models was tested by an $F$ test where $F=\left(\right.$ factor SSE $/ \mathrm{df}_{\text {fac }}$ )/ (null model SSE/ $\mathrm{df}_{\text {SSE }}$ ), and $\mathrm{df}_{\mathrm{fac}}$ and $\mathrm{df}_{\text {SSE }}$ are the $\mathrm{df}$ of the factor and modeled sum of squares, respectively.

Based on results of the covariate analysis, factors that were significant and deemed to have a large influence on automated estimates were used to subset the validation data set. Images that were affected by one or more of these factors were excluded and the relationship between hand counts and automated estimates modeled. For each field plot sampled, quadrat means were calculated for hand samples and automated estimates and modeled by regression.

\section{Results}

System DeVelopment. Hue values of pyrethrum disc florets obtained ranged from 16 to 113 , with a median of 34 (Fig. 2). Saturation and brightness ranged from 12 to 255 $($ median $=221)$ and 53 to 255 (median $=185)$, respectively. For each parameter, the $1 \%$ and $99 \%$ quantiles of observed values were selected as the lower and upper limits for flower recognition. This resulted in threshold limits of 25 to 45,180 to 255 , and 60 to 255 for hue, saturation, and brightness, respectively.

Initial estimates of particle size indicated a maximum of $5000 \mathrm{px}^{2}$. With this upper size limit combined with circularity limits of 0 and 1 , the median percentage error of automated estimates was minimized using a lower size limit of $50 \mathrm{px}^{2}$ (3.76\%; Fig. 3A). With this lower size limit, decreasing the maximum size limit to $4500 \mathrm{px}^{2}$ did not improve the median error, while further reductions in the maximum size increased the median errors obtained. Increasing the maximum limit to infinity did not alter error estimates relative to the $5000-\mathrm{px}^{2}$ limit. Similarly, median error was minimized when the extreme circularity limits of 0 and 1 were used (Fig. 3B). No differences were observed in the distribution of errors between the lower limits of 0 and 0.1 . However, further increases in the lower limit increased the observed error. Based on these results, particle analysis size and circularity limits were set at 50 to $5000 \mathrm{px}^{2}$ and 0 to 1 , respectively, for further analyses.

The relationship between automated and manual estimates of flower numbers was described by the equation (Fig. 4A):

\section{Estimated number of flowers $=0.884$}

$\times$ manual estimate of flower number $+30.8$

The intercept did not differ significantly from $0(P=0.068)$. However, the slope parameter was significantly less than $1(P=0.0009)$. Good concordance was estimated between manual and automated estimates ( $\rho c=0.941,95 \% \mathrm{CI}=0.878-0.965)$, with a corrected bias estimate of 0.977 $(95 \%$ CI $=0.945-0.989)$. Small scale $(1.089,95 \% \mathrm{CI}=1.027-1.257)$ and location $(0.198,95 \%$ CI $=0.136-$ 0.297 ) shifts were estimated.

MODELING SAMPLE SIZE. Goodnessof-fit tests rejected the fit of the
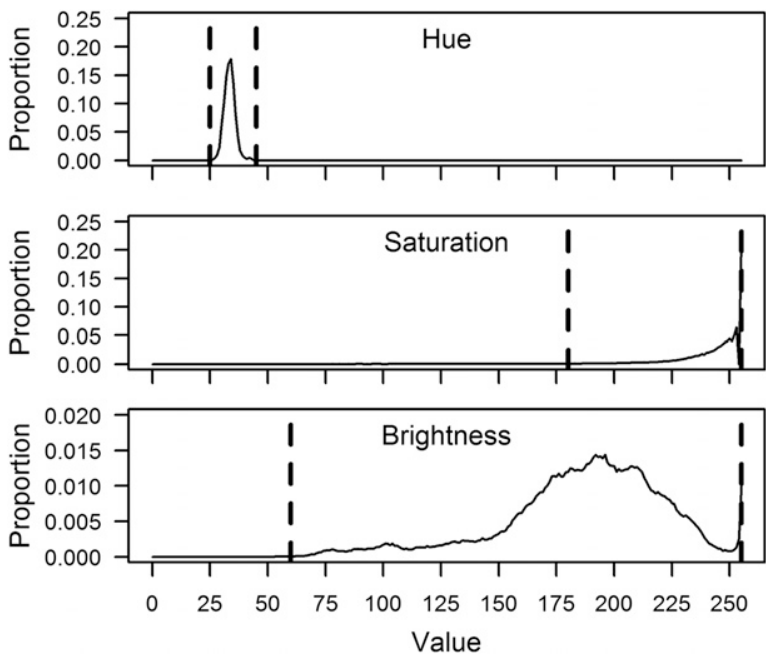

Fig. 2. Hue, saturation, and brightness pixel profiles of manually selected disc florets from field-collected images of pyrethrum flowers. Parameters were measured on scales of 0 to 255. Vertical dashed lines indicate upper (right) and lower (left) limits for each parameter used for color-thresholding of images. 
Poisson distribution $(P<0.001)$, while accepting the fit of the negative binomial distribution $(P \geq 0.39)$ for all 68 plots. The log-likelihood ratio statistic rejected the Poisson distribution in favor of the negative binomial distribution for all plots. Taylor's
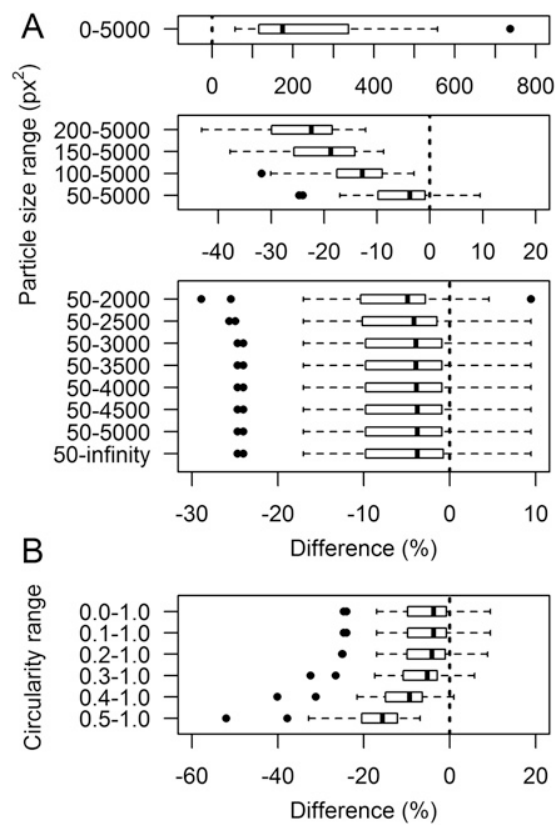

Fig. 3. Error distributions obtained during optimization of the pixel size in square pixels [ $\left.\mathrm{px}^{2}(\mathrm{~A})\right]$ and circularity (B) parameters for the "Analyze

Particles" function of ImageJ. Boxplots representing the percentage errors of automated estimates relative to manual estimates of visible pyrethrum flowers within digital images. Thick lines of individual boxes represent the median percentage difference, while box borders indicate the $25 \%$ and $75 \%$ percentiles. Whiskers represent $1.5 \times$ the interquartile range. Dots represent outliers.
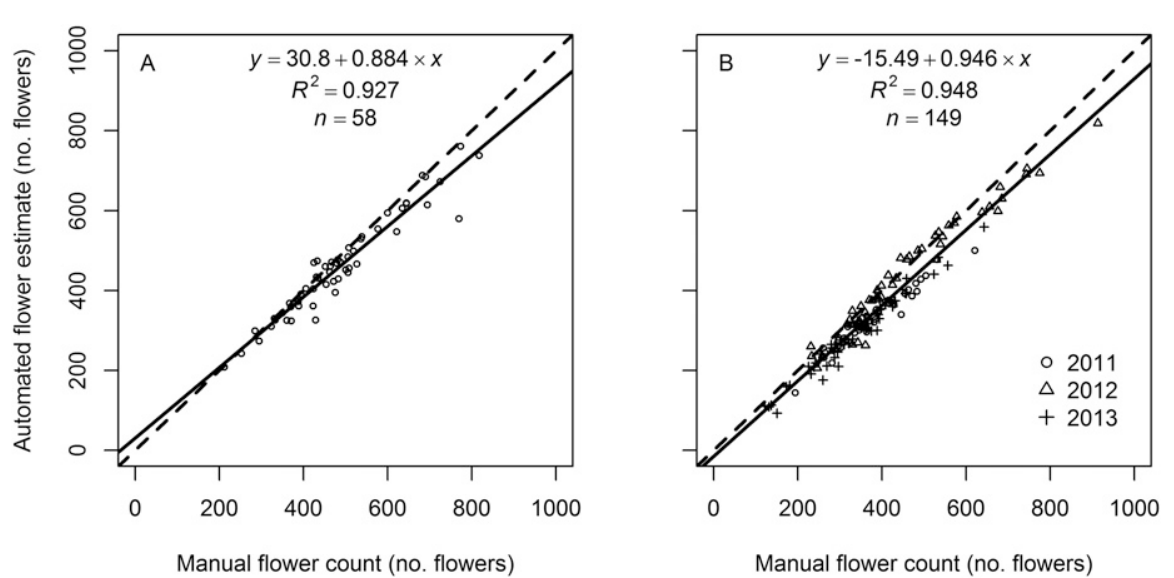

Fig. 4. Relationships between automated and manual estimates of visible pyrethrum flowers using images used for system development (A) and validation (B).

power law indicated a weak relationship between plot mean and variance, with estimated slope and intercept parameters of 0.22 and 3.27 , respectively. The predictive power of this model was poor, with a coefficient of determination of 0.009 . Thus, this relationship was not used to develop a predictor of required sample size.

Required sample size to achieve a set $\mathrm{CV}$ was calculated for each plot individually using the related estimate of $k$ from the negative binomial distribution (Fig. 5). To achieve a CV of 0.1 , sample sizes ranged from 0.56 to 13.7, with a median of 2.59. Ninetyfive percent of estimated samples sizes were 7.18 or less. Decreasing the target $\mathrm{CV}$ to 0.05 resulted in a median required sample size of 10.37 (range 2.23 to 54.69 ).

Estimated sample size increased with decreasing flower number (Fig. $5)$. This relationship was examined for a CV of 0.1 , where model deviance was minimized using the power model (91.96), relative to the exponential (98.41) and linear models (206.04). Under the power model, sample size $=3,628,468 \times($ mean flower count per plot $)^{-2.247}$. Based on the $95 \%$ quantile to achieve a targeted CV of at least 0.1 , eight random samples ( 7.18 rounded up) were selected from each validation plot for each of 1000 bootstrap replicates. Linear regression of the median of bootstrap replicates indicated a $1: 1$ response of estimated mean flower count per plot to increases in true mean flower count per plot. Slope $(0.9996)$ and intercept $(-0.001)$ estimates of this relationship were not significantly different from

Hortlechnology $\cdot$ October 2015 25(5)
$1(P=0.878)$ and $0(P=0.999)$, respectively, with a coefficient of determination of 0.999 (Fig. 6A). Concordance estimates also indicated

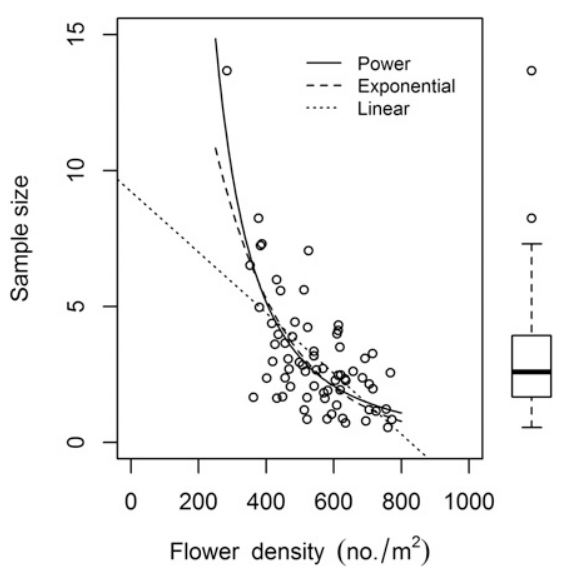

Fig. 5. Relationship between mean pyrethrum flower density $(\bar{F})$ and estimated sample size $(N)$ required to achieve a CV of 0.1 . Sample size estimated using the equation $N=(1 / \bar{F}+1 / \hat{k}) \cdot\left(1 / \mathrm{CV}^{2}\right)$ where $\hat{k}$ is the dispersion parameter for each site estimated by fitting the negative binomial distribution. Barplot on right represents the distribution of all sample size estimates. Median is indicated by the thick central line, while borders of box signify the $25 \%$ and $75 \%$ percentiles. Whiskers represent $1.5 \times$ the interquartile range. Open circles indicate outliers; 1 flower $/ \mathrm{m}^{2}=0.0929$ flower $/ \mathrm{ft}^{2}$.

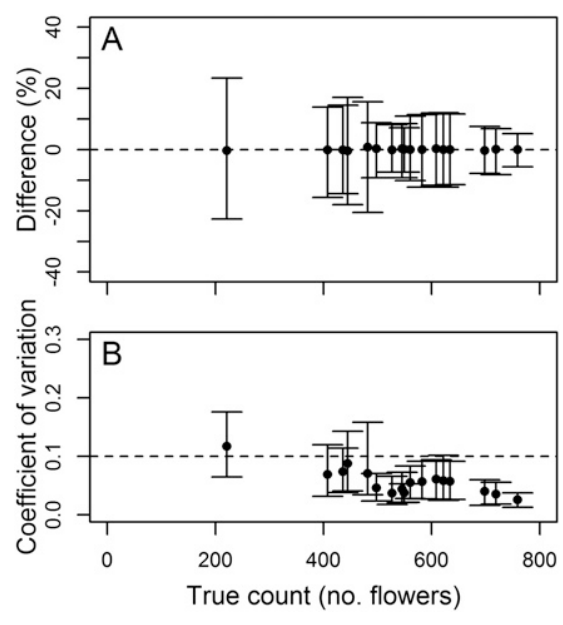

Fig. 6. Percentage difference from true pyrethrum flower density (A) and achieved CV (B) by randomly sampling eight images from a full set of $\mathbf{5 0}$ images for each of 17 field plots. Error bars represent the $2.5 \%$ and $\mathbf{9 7 . 5 \%}$ percentiles from 1000 random bootstrap samples. 
a perfect relationship between the two parameters $(\rho c=1.000,95 \%$ $\mathrm{CI}=1.000-1.000 ; \mathrm{C}_{\mathrm{b}}=1.000,95 \%$ $\mathrm{CI}=1.000-1.000)$ with no scale $(1.000,95 \%$ CI $=0.996-1.008)$ or location $(-0.002,95 \% \mathrm{CI}=-0.009-$ 0.003 ) shifts. When the estimated mean flower count was regressed against the true mean flower count within each individual bootstrap replicate, estimated intercept and slope parameters differed from 0 and 1,31 $(3.1 \%)$ and $28(2.8 \%)$ times $(P<$ $0.05)$, respectively. Across individual plots, 16 of 17 plots achieved a median $\mathrm{CV}$ of 0.1 or better with eight random samples (Fig. 6B). The upper 95\% CI for achieved CV was less than 0.1 , for 11 of 17 plots.

System validation. Modeling the response of automated flower count to manual flower count produced slope $(0.946 ; P=0.0036)$ and intercept $(-15.49 ; P=0.040)$ estimates that differed significantly from 1 and 0 , respectively. Slope $(P=$ $0.0009)$ and intercept $(P<0.001)$ estimates differed from those obtained during system development. The estimated concordance correlation coefficient $\left(\rho_{c}=0.936,95 \% \mathrm{CI}=0.914-0.952\right)$ was consistent with the estimate obtained during system development, as was the estimate of corrected bias $\left(\mathrm{C}_{\mathrm{b}}=0.961,95 \% \mathrm{CI}=0.946-0.972\right)$. Analysis estimated a location shift $(0.283,95 \% \mathrm{CI}=0.238-0.337)$ indicating an underestimation of flowers from automated flower counts relative to manual flower counts. No scale shift was estimated $(1.029,95 \% \mathrm{CI}=$ 0.989-1.067).

Modeling of the response of automated flower counts to handsampled flower counts (Fig. 7A) resulted in a slope $(0.325)$ that differed from $1(P<0.001)$, while the intercept (51.5) was greater than $0(P=0.011)$. Covariate analysis indicated that the factors Maturity, Lodging, and Shadows all had a significant effect on the intercept $(P \leq$ 0.037; Table 1) and slope ( $P \leq$ $0.015)$. The occurrence of shadows and lodging reduced both intercept and slope estimates, while mature flowers increased estimates of both these parameters. The influence of Lodging and Maturity was observed to be larger than Shadows.

Exclusion of images affected by mature flowers and/or lodging produced a subset of 100 images
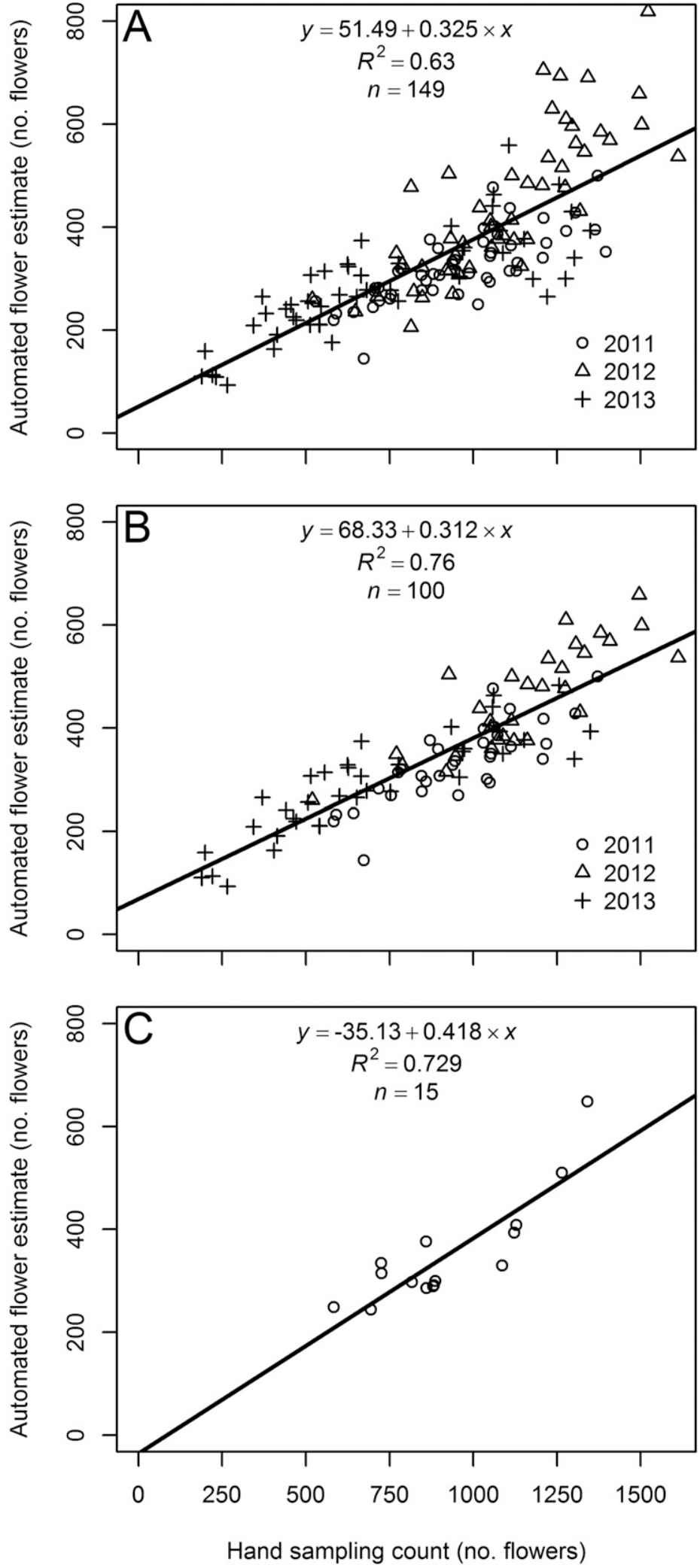

Fig. 7. Relationship between automated estimates and hand-harvested counts of pyrethrum flower numbers from five pyrethrum fields each from 2011, 2012, and 2013, with 10 sampling units per field (one missing sampling unit in 2013); (A) all sampling units; (B) sampling units affected by lodging and maturity of flowers excluded; and (C) field means. 
Table 1. Covariate analysis of the potential error factors of shadowing, lodging, rain, and flower maturity on slope and intercept estimates from the relationship between automated estimates and hand-harvested counts of pyrethrum flower numbers.

\begin{tabular}{|c|c|c|c|c|c|c|c|c|c|c|c|c|c|c|}
\hline \multirow[b]{2}{*}{ Model } & \multirow[b]{2}{*}{$\mathrm{df}_{S S E}^{\mathrm{z}}$} & \multirow[b]{2}{*}{$\mathrm{df}_{\mathrm{fac}}^{\mathrm{z}}$} & \multicolumn{6}{|c|}{ Intercept $^{\mathrm{y}}$} & \multicolumn{6}{|c|}{ Slope } \\
\hline & & & Est. 0 & Est. 1 & SSE & Difference & $F$ & $P$ & Est. 0 & Est. 1 & SSE & Difference & $F$ & $P$ \\
\hline Base & 147 & & & & $886,912.1$ & & & & & & $886,912.1$ & & & \\
\hline +Lodging & 146 & 1 & 74.83 & 1.14 & $733,526.9$ & $153,385.2$ & 30.53 & $<0.001$ & 0.34 & 0.25 & $675,948.2$ & $152,041.9$ & 38.59 & $<0.001$ \\
\hline +Rain & 146 & 1 & 51.31 & 57.05 & $886,663.1$ & 249.0 & 0.041 & 0.840 & 0.32 & 0.34 & $884,830.7$ & $4,577.1$ & 0.93 & 0.338 \\
\hline +Maturity & 146 & 1 & 45.19 & 185.9 & $652,601.8$ & $234,310.3$ & 52.42 & $<0.001$ & 0.30 & 0.46 & $566,593.5$ & $320,318.6$ & 82.54 & $<0.001$ \\
\hline
\end{tabular}

${ }^{\mathrm{z}} \mathrm{df}_{\mathrm{SSE}}=\mathrm{df}$ for the base model SSE; $\mathrm{df}_{\text {fac }}=\mathrm{df}$ for factor included as a covariate of either the slope or intercept term.

yst. 0 = estimated slope or intercept where error factor is coded as absent; Est. 1 = estimated slope or intercept where error factor is coded as present; SSE = sum of squares error; Difference = difference in SSE between the base model and the alternative, the base model with relevant factor included as a covariate in either the slope or intercept term;

$F=F$ test for significance of the difference in SSE between models, where $F=\left(\right.$ factor SSE $\left./ \mathrm{df}_{\text {fac }}\right) /\left(\right.$ model SSE$\left./ \mathrm{df}_{\mathrm{SSE}}\right) ; P$ represents the probability of significance of this test.

(Fig. 7B). A linear regression of true mean flower count and estimated mean flower count among these images had slope and intercept parameters of 0.312 and 68.33 , respectively. Neither the intercept $(P=0.331)$ nor slope $(P=0.477)$ estimates from the subset of images differed from the full validation data set. A linear regression of mean flower counts by image analysis and mean flower count determined by hand counts had slope $(0.418)$ and intercept $(-35.13)$ estimates that did not differ significantly from the full validation data set $(P \geq$ 0.209; Fig. 7C).

\section{Discussion}

Image analysis enabled the development of a system for in situ pyrethrum flower number estimation. Automated recognition of flowers consistently detected $88 \%$ of the flowers detected by manual recognition. When compared with hand collection and physical counting of flowers from sampling units, the system recognized $\approx 32 \%$ of all flowers collected. This relationship was consistent across all flower densities encountered in this study, which encompassed all typical densities of those in commercial fields. This discrepancy may be at least in part due to the three-dimensional architecture of the canopy due to variability in stem height. Reduced flower densities appeared associated with reduced densities in the horizontal plane, but relatively consistent degrees of vertical canopy density. Although it was not observed in this study, the potential for flower number to be sufficiently high that vertical density of the canopy increases due to a saturated horizontal plane cannot be discounted. Estimates obtained from very high density crops should therefore be treated with caution.

An important requirement for the implementation of this system for estimating flower number is the ability to provide accurate estimates that are representative of the sampling area. Measurements of the spatial distribution of flowers in this study indicated a tendency for spatial aggregation. Taylor's power law failed to adequately describe the relationship between flower density and aggregation, and thus the required number of samples for a set level of accuracy (Madden et al., 2007). However, when degree of aggregation was accounted for within individual plots, there was a general trend of increasing samples sizes required for decreasing flower density. Thus, to determine the theoretically optimal number of sampling units for a given plot a presampling estimation of density and aggregation are required. Given the relative speed of field sampling in our system, oversampling to ensure an accuracy threshold is likely to be more expedient, since only eight sampling units were needed to achieve a CV of 0.1 in a plot area of $\approx 600 \mathrm{~m}^{2}$.

Observations of images collected for system validation indicated several sources of significant, albeit relatively modest, errors. Advanced maturity of flowers resulted in senescence of their ray florets and overestimation of flower numbers. This was problematic because more mature crops have a higher risk of lodging and the change in color with maturity from yellow to brown make the settings of the image processing inoperable. Lighting and environmental conditions were also observed to influence flower number estimates. Under highcontrast (i.e., sunny) conditions, shadows within images masked flowers from the "Analyze Particles" function. Although rain did not significantly affect the results, its influence cannot be fully discounted. It is advisable, where possible, to avoid using image analysis where lighting, weather, and crop conditions are most likely to result in estimation errors. As an additional control, over sampling with censoring of poor quality images before analysis should be employed.

\section{Conclusions}

The key advantages of this automated system are the speed and ease of image collection, and subsequent flower estimation. This enables a much greater plot area to be sampled for yield estimation than manual sampling. Given the inherent spatial aggregation of pyrethrum flowers quantified here, the ability of this system to sample larger numbers of points within a plot area is critical for obtaining accurate estimates of flower density. Subsamples of flowers would still need to be manually removed for estimation of pyrethrin ester content by chemical analyses if this was also required. Nonetheless, the image analysis approach will still greatly improve the speed of estimating an important component of pyrethrum yield. With the steady improvements in digital imaging and remote sensing abilities, the system also could be readily scaled up to nondestructively estimate the number of flowers over larger areas and further eliminate sampling errors. While the system developed here was specifically for pyrethrum, it could be easily modified for any crop where the color and size of a flower or other plant tissue can be differentiated from the background canopy. 


\section{Literature cited}

Bechar, A., S. Gan-Mor, Y. Vaknin, I. Shmulevich, B. Ronen, and D. Eisikowitch. 1997. An image-analysis technique for accurate counting of pollen on stigmas. New Phytol. 137:639-643.

Canty, A. and B. Ripley. 2015. boot: Bootstrap R (S-Plus) Functions. R package. Version 1.3-16. 3 Aug. 2015. <http://CRAN.R-project.org/ package $=$ boot $>$.

Casida, J.E. and G.B. Quistad. 1995. Pyrethrum flowers: Chemistry, toxicology and uses. Oxford Univ. Press, New York, NY.

Costa, C.M. and S.A. Yang. 2009. Counting pollen grains using readily available, free image processing and analysis software. Ann. Bot. (Lond.) 104:1005-1010.

Ghali, I.E., G.L. Miller, G.L. Grabow, and R.L. Huffman. 2012. Using variability within digital images to improve tall fescue color characterization. Crop Sci. 52:2365-2374.

Henry, A., V.R.P. Gowda, R.O. Torres, K.L. McNally, and R. Serraj. 2011. Variation in root system architecture and drought response in rice (Oryza sativa): Phenotyping of the OryzaSNP panel in rainfed lowland fields. Field Crops Res. 120:205-214.

Li, J., M.A. Jongsma, and C.Y. Wang. 2014. Comparative analysis of pyrethrin content improvement by mass selection, family selection and polycross in pyrethrum [Tanacetum cinerariifolium (Trevir.) Sch.Bip.] populations. Ind. Crops Prod. 53:268-273.

Lin, L.I. 1989. A concordance correlation coefficient to evaluate reproducibility. Biometrics 45:255-268.

Macdonald, W.L. 1995. Pyrethrum flowers-Production in Australia, p. 55-66. In: J.E. Casida and G.B. Quistad (eds.). Pyrethrum flowers: Chemistry, toxicology and uses. Oxford Univ. Press, New York, NY.
Madden, L.V., G. Hughes, and F. van den Bosch. 2007. The study of plant disease epidemics. Amer. Phytopathol. Soc., St. Paul, MN.

Pethybridge, S.J., P. Esker, P. Dixon, F. Hay, T. Groom, C. Wilson, and F.W. Nutter. 2007. Quantifying loss caused by ray blight disease in Tasmanian pyrethrum fields. Plant Dis. 91:1116-1121.

Pethybridge, S.J., D.H. Gent, L. Hingston, and P. Frost. 2014. Quantifying the effects of uniconazole on growth and yield of pyrethrum in Australia. N. Z. J. Crop Hort. Sci. 42:50-59.

Pethybridge, S.J., F. Hay, P. Esker, T. Groom, C. Wilson, and F.W. Nutter. 2008a. Visual and radiometric assessments for yield losses caused by ray blight in pyrethrum. Crop Sci. 48:343-352.

Pethybridge, S.J., F.S. Hay, P.D. Esker, D.H. Gent, C.R. Wilson, T. Groom, and F.W. Nutter. 2008b. Diseases of pyrethrum in Tasmania: Challenges and prospects for management. Plant Dis. 92:1260-1272.

Pethybridge, S.J., F.S. Hay, C.R. Wilson, and T. Groom. 2005. Development of a fungicide-based management strategy for foliar disease caused by Phoma ligulicola in Tasmanian pyrethrum fields. Plant Dis. 89:1114-1120.

R Core Team. 2015. R: A Language and Environment for Statistical Computing. Version 3.0.2. 3 Aug. 2015. <http:// www.R-project.org $>$.

Rao, B.R.R., S.P. Singh, and E. Rao. 1983. N,P and $\mathrm{K}$ fertilizer studies in pyrethrum (Chrysanthemum cinerariifolium). J. Agr. Sci. 100:509-511.

Salardini, A.A., K.S.R. Chapman, and R.J. Holloway. 1994a. Effect of basal and sidedressed phosphorous on the achene yield and pyrethrins concentration in the achenes of pyrethrum (Tanacetum cinerariifolium) and on soil and plant phosphorous. Austral. J. Agr. Res. 45:231-241.
Salardini, A.A., K.S.R. Chapman, and R.J. Holloway. 1994b. Effect of potassium fertilization of pyrethrum (Tanacetum cinerariffolium) on yield, pyrethrins concentration in dry achines and potassium concentration in soil and plant tissues. Austral. J. Agr. Res. 45:647-656.

Schneider, C.A., W.S. Rasband, and K.W. Eliceiri. 2012. NIH Image to ImageJ: 25 years of image analysis. Nat. Methods 9:671-675.

Severini, A.D., L. Borras, and A.G. Cirilo. 2011. Counting maize kernels through digital image analysis. Crop Sci. 51:27962800 .

Stevenson, M., T. Nunes, C. Heuer, J. Marshall, J. Sanchez, R. Thornton, J. Reiczigel, J. Robison-Cox, P. Sebastiani, P. Solymos, K. Yoshida, and S. Firestone. 2015. epiR: Tools for the Analysis of Epidemiological Data. R Package. Version 0.9-62. 3 Aug. 2015. <http:// CRAN.R-project.org/package $=$ epiR $>$.

Tajima, R. and Y. Kato. 2013. A quick method to estimate root length in each diameter class using freeware ImageJ. Plant Prod. Sci. 16:9-11.

Taylor, L.R. 1961. Aggregation, variance and mean. Nature 189:732-735.

Venables, W.N. and B.D. Ripley. 2002. Modern applied statistics with $S$. Springer, New York, NY.

Wanjaia, B.W. 1990. Influence of farmyard manure, phosphatic and nitrogenous fertilizers on pyrethrum production. Pyrethrum Post 18:55-60.

Williams, K., J. Munkvold, and M. Sorrells. 2013. Comparison of digital image analysis using elliptic Fourier descriptors and major dimensions to phenotype seed shape in hexaploid wheat (Triticum aestivum L.). Euphytica 190:99-116.

Zito, S.W., R.G. Zieg, and E.J. Staba. 1983. Distribution of pyrethrins in oil glands and leaf tissue of Chrysanthemum cinerariaefolium. Planta Med. 47:205207. 